\title{
Distancia sin distancia \\ El oxímoron como lugar de encuentro de lo humano. Una mirada a la modalidad de educación a distancia desde la didáctica no parametral
}

\author{
No Distance Distance \\ The oxymoron as a meeting place of the human. A look at the form of distance education \\ from teaching not parametral
}

\author{
Cesar Augusto Gómez Londoño ${ }^{1}$ \\ Universidad Central del Valle del Cauca \\ Valle del Cauca, Colombia \\ cesarago@hotmail.com \\ ORCID: orcid.org/0000-0002-6192-4835 \\ Gustavo Adolfo Cárdenas Messa ${ }^{2}$ \\ Universidad Central del Valle del Cauca \\ Valle del Cauca, Colombia \\ gcardenas@uceva.edu.co \\ ORCID: http://orcid.org/0000-0002-7571-9023
}

Recibido: 11 junio 2015 Aceptado: 16 junio 2016 Corregido: 19 abril 2016

\begin{abstract}
Resumen: La investigación en educación es un escenario de enunciación, fundamentalmente un ejercicio de lenguaje que nos permite poner en escena una propuesta tanto de adaptación teórica como de reflexión sobre un tópico, en este caso, la modalidad de educación a distancia de la Unidad Central del Valle UCEVA, mirada desde la perspectiva de la didáctica no parametral. La propuesta se basa en la aproximación al ejercicio didáctico del personal docente del programa de Licenciatura en Educación Básica con énfasis en Ciencias Sociales, en la modalidad a distancia. Realizar una caracterización de las prácticas educativas desde sus perspectivas respecto al proceso mismo de enseñanza/aprendizaje, de manera puntual, desde la perspectiva de las implicancias que tiene la modalidad a distancia en las relaciones interhumanas de los sujetos de formación y sus maestros y maestras, las mediaciones que les permiten acercamientos o que propician escenarios rupturales de las interacciones humanas en el proceso didáctico. Se usó una metodología de diálogo estructurado, basado en instrumentos como el grupo focal, la entrevista y el análisis meta narrativo de los resultados. Se encontró como resultado que las prácticas actuales en este modelo educativo no privilegian la formación humana, ni las interacciones entre estudiantes y docentes, más allá del transmisionismo pedagógico.
\end{abstract}

Palabras clave: Didáctica, educación a distancia, docente, estudiante, enseñanza no tradicional.

1 Ingeniero Industrial, Universidad Central del Valle; Diplomado en Estrategias didácticas para el desarrollo del estudio independiente y para la elaboración de material educativo de apoyo, Coruniversitec; docente de educación media (Colegio San Juan Bosco, Tuluá Valle), docente universitario (Universidad Central del Valle del Cauca, Universidad Autónoma de Occidente), ha sido docente de instituciones como Uniciencia, Coruniversitec, EAN. Director y jurado de trabajos de grado en diferentes programas académicos. Magister en Educación (Universidad Católica de Manizales).

2 Licenciado en Literatura (Universidad del Valle), Profesional en Ciencias de la Información y la Documentación (Universidad del Quindío), Diplomado en Ingeniería de Formación (société française d'exportation des ressources éducatives, SFERE), docente universitario (Universidad Central del Valle del Cauca), director y jurado de trabajos de grado universitarios en diversos programas académicos, ha sido docente en instituciones como: Universidad del Valle, Universidad Tecnológica de Pereira, Instituto de Educación Técnica Profesional de Rodanillo, Magister en Educación (Universidad Católica de Manizales). 


\begin{abstract}
Educational research is a scenario of enunciation. It is fundamentally an exercise of language that allows us to stage a theoretical and reflective proposal about a topic, in this case, distance-education model at Unidad Central del Valle (UCEVA), seen from the perspective of nonparametric didactics. The proposal is based on the approach of the professors in the academic program of Bachelor Degree in Basic Education with Emphasis on Social Science to the didactic exercise, in the distance-education model. Perform a characterization of their educational practice from their perspectives, regarding the teaching/learning process itself, specifically, from the perspectives of the implications that the distance-education model has about students and professors' interpersonal relationships, their mediations that let them to approach each other or bring them rupturing scenarios of human interactions in the didactic process. A structured-dialogue methodology was used, based on instruments as focus group, interview and meta-narrative analysis of the results. It was found as results that current practices in this educational model do not privilege the human formation, or the teacher-student interaction further than pedagogical transmissionism.
\end{abstract}

Keywords: Didactics, distance education, teacher, student, nontraditional education.

La educación a distancia es un Modelo en el cual intervienen los medios impresos, mecánicos y electrónicos para facilitar la relación profesor estudiante y promover la interacción para superar la separación física entre ambos.

\title{
Borge Holmberg
}

El presente estudio ${ }^{3}$ buscó una aproximación comprensiva a la realidad didáctica en la cual se enmarca la práctica docente en la modalidad de educación a distancia, en el contexto de la Unidad Central del Valle del Cauca, universidad colombiana con programas con carácter de semipresencialidad, está agrupada en un departamento de educación a distancia. A esta oficina está adscrita la Licenciatura en Educación Básica con Énfasis en Ciencias Sociales.

Se pretendió hacer una caracterización de las prácticas didácticas de sus docentes, específicamente desde la perspectiva del encuentro, del acercamiento humano, enmarcado en un modelo que propicia más el distanciamiento y la educación trasnmisionista. Esta caracterización estuvo mediada por el filtro conceptual de la didáctica no parametral, concepto abordado por Estela Beatriz Quintar y Hugo Zemelman, entre otras teóricas teóricos contemporáneos. En este sentido, Quintar plantea: "Lo didáctico ha quedado entrampado en parámetros que lo determinan como recetario de modos de dar clase" Este reconocimiento implica aceptar un estatismo atávico en los procesos de enseñanza/aprendizaje, reconocer que pese a la constante evolución del discurso y de las teorías educativas, en la práxis hay todavía caminos no recorridos hacia una mayor humanización del acto educativo: "pensar una didáctica no parametral como espacio que contribuya a reedificar un campo de acción profundamente humano" (1998, p. 20).

Dos categorías serán leit motiv a lo largo de este artículo: Maestros y maestras, y estudiantes. En medio de un amplio abanico de posibilidades (docente, profesor, instructor,

3 El presente artículo es derivado de la tesis de maestría, Distancia cercana, de la UCM, recuperable en el enlace http://repositorio.ucm. edu.co:8080/jspui/bitstream/handle/10839/799/Gustavo\%20Adolfo\%20Cardenas $\% 20$ Messa.pdf?.sequence=1 
enseñante-alumno, aprendiente, discente) hemos elegido estas dos categorías por considerarlas cercanas a la intencionalidad comunicativa de esta obra: La humanización de lo humano en la modalidad de educación a distancia. Un maestro o maestra que no solo educa sino que tiene cercanías afectivas, emocionales con sus estudiantes y con el saber. Un estudiante o una estudiante como aquella persona que estudia, es decir, que tiene intereses cognoscitivos, pero también se reconoce como sujeto, como par en el acto educativo. "¿Qué significa educar estas personas? Fernando Savater dice que educar es creer en la perfectibilidad humana y que el sentido de la educación es transmitir el amor intelectual a lo humano" (Quintar, 1998, p. 106).

Para dar inicio formal a este camino, visibilizaremos entonces nuestro escenario, donde esta obra cobró vida más allá del discurso. La educación a distancia en la Unidad Central del Valle UCEVA, de Tuluá Valle, una institución universitaria pública, colombiana, en la cual la educación a distancia ha sido un modelo que ha respondido al concepto de convenio interuniversitario. La Universidad Tecnológica y Pedagógica de Colombia UPTC de Tunja y la UCEVA han venido trabajando mancomunadamente en la formación de profesionales en tecnologías y licenciaturas en educación básica, con la modalidad a distancia. En este escenario, estudiantes y maestros y maestras tienen cuatro encuentros presenciales de 2.5 horas cada uno, para un total de 10 horas de encuentro, durante un mes. Las interacciones entonces se dividen en 10 horas de presencialidad y 30 de trabajo autónomo guiado, sin jornadas programadas de encuentro virtual entre sí.

En medio de esta realidad educativa hay otra realidad: La distancia que se transforma en distanciamiento, en lejanía, ni siquiera mediada por los recursos tecnológicos. Es aquí donde esta propuesta pretendió entender cómo se maifiestan las interacciones de cercanía, de encuentro humano, o de distanciamiento. Si pensamos que "la educación a distancia es el tipo de método de instrucción en que los conductas docentes acontecen aparte de las discentes" (Moore, 1972 citado por García, 1991, p. 6), esto implica que la modalidad a distancia es fundamentalmente instruccional. En un modelo híbrido como el del territorio objeto de este estudio, donde existe una mínima presencialidad, es importante determinar si esta presencialidad opera en el imaginario docente como elemento potenciador de las interacciones humanas necesarias en un proceso educativo no instructivo, o si la noción de educación a distancia se constituye en un condicionante de perpetuación del modelo eminentemente transmisionista.

Ahora, pensar en la acción didáctica del maestro o maestra vale la pena porque implica pensar en la educación no solo como una acción mecánica transmisionista, migratoria de saberes, sino como una acción de interacciones humanas, de relaciones entre seres humanos que no solo comparten conocimientos, sino experiencias, vivencias, sentimientos, apasionamientos. Finalmente, la educación es un compartir saber/hacer/ser y, en esta triada, el compartir el ser significa implicarse e implicar a la otra persona. No de otra forma se logra propiciar el escenario de aprendizaje significativo (Ausubel, 1981). Esta implicancia representa un lugar común de encuentro, de reconocimiento de la otredad, y de compartir con el otro individuo los propios apasionamientos, dudas y convicciones; es aquí, en este lugar comùn, donde es posible el aprendizaje.

Y se hace esencial hablar de didáctica, hoy cuando la educación se enfrenta a una dicotomía práctica: El enfrentamiento entre los arraigos en las prácticas educativas docentes, 
y las nuevas mediaciones propias de la tecnología y de los nuevos discursos educativos. En este momento, las prácticas didácticas se hacen esenciales en el momento de ofrecer al estudiantado un referente cognoscitivo, y un referente cultural. Así, la mediación que el maestro o maestra hace deja de ser puramente instrumental y se convierte en un compartir de su ideario frente a la cultura. Así lo entiende Margarita Victoria Gómez cuando plantea que "modernizar la escuela y actualizar a los profesores para actuar en un mundo cambiante en lo cultural, social y laboral, se torna sin lugar a dudas imperioso" (2001, p. 205).

Hugo Zemelman (2006) nos dice sobre la didáctica no parametral: "el pensamiento que invita a la deconstrucción y construcción libertaria de nuestra realidad como espacio posible de ser permanentemente transformado asumiendo esta posibilidad como opción histórica" ( $p$. 108). A partir de esta premisa se plantea un recorrido por algunas obras que han abordado este mismo interés de conocimiento, desde ópticas tan variadas que representan en sí mismas el complexus de la relación del sujeto con el conocimiento, por la mediación del acto educativo.

Saa y Garcés (2012, p. 14) reconocen que "sin un pensamiento crítico, problematizador y transformador, que provoque la reflexión sobre el ser que educamos y sobre el sentido por el cual se educa, estos desafíos como opción histórica de nuestra comunidad educativa se dificultan". Este reconocimiento es una aproximación a la noción fundante de esta propuesta, la educación desde la mirada de lo humano, trascendiendo la instrumentalización, el proceso migratorio de saberes que no constituyen en sí mismos aprendizajes. Reconocer a la otra persona, la otredad, es la manera como la educación obra sobre la conciencia histórica del sujeto, sobre su condición de sujeto cultural y social, con el reconocimiento de sus dimensiones humanas y su naturaleza intelectual.

La teorética educativa ha sufrido notables transformaciones y diversas vertientes que la ubican como fenómeno cognitivo, social o interaccional, pero en la praxis educativa en general no se evidencia una evolución del pensamiento educativo individual hacia una mirada de la posmodernidad como un entramado histórico que requiere no solo otras teorías, sino otras prácticas, otras interacciones.

En este mismo sentido las mencionadas autoras plantean "la didáctica emergente como instrumento que permite en los procesos pedagógicos potenciar el pensamiento complejo en el sujeto educable" (Saa \& Garcés, 2012, p. 29), como reconocimiento del proceso transformador de la educación a través de un instrumento vertebral: La didáctica, no como la ludización o estandarización metodológica del acto educativo, sino como la inserción de este en contexto del sujeto educable, como lo plantea Zambrano Leal (2001). Mirar la mirada del sujeto educable es mirar su condición humana, y hacer de la didáctica un lugar común, no solo del lenguaje, sino de la condición de humanidad, expresada en el afecto, en los actos de amor por el otro individuo, actos didácticos de amor. Potenciar el pensamiento complejo en el sujeto educable es, fundamentalmente, darle las condiciones necesarias para que pueda construir imaginarios de realidad más globales, menos fragmentados.

El encuentro con el sujeto educable desde su humanidad, desde el intercambio de experiencias, y desde modelos de acercamiento y relación diversos, interestructurantes (De Zubiría, 2006), dialogantes, humanizantes, es pues, condición necesaria en un proceso educativo no instructivo. Una persona docente que no se reconoce como intelectual, que no aborda su hacer 
como un ejercicio de resignificación del mundo, sino como una repetidora de viejas fórmulas, hoy disponibles en la web, no puede propiciar en sus estudiantes el pensamiento crítico, divergente, que dilucide la realidad a través del filtro de sus propias experiencias, pero también, de sus aprendizajes hechos gracias a la mediación del maestro o maestra.

Buitrago, Torrez y Hernández (2009, p. 7) enfocan su tesis de maestría en el reconocimiento de los saberes de estudiantes como insumo necesario en el proceso de enseñanza: "Sostenemos que favorecer un balance adecuado entre contenidos y saberes de los estudiantes, es posible al insertar, en el proyecto de aula, una secuencia didáctica, de forma que se permita un acercamiento específico al contenido particular". Ello es una buena forma de decir que reconocer los saberes del sujeto educable implica reconocerlo como sujeto, desde el ámbito cognitivo, como puerta de entrada a la formulación de nuevas didácticas, de nuevas esferas relacionales.

Para las autoras, el concepto de secuencia didáctica se constituye en la ruta gnoseológica, metódica y pragmática desde la cual se articula el acto de aula, y la relación entre docente, contenidos programáticos, estudiantes y su entorno.

Esta apuesta pretende que el estudiante aprenda, no solo el conocimiento teórico, sino a vivir dentro de una comunidad, resolviendo los problemas que se le presentan a diario, a través de espacios de participación democrática y reflexiva, definiendo nuevas formas de relación entre los sujetos, y entre estos con el conocimiento y la cultura. (Buitrago, Torres, \& Hernández, 2009, p. 26)

De esta forma, el planteamiento vertebral de esta obra es reconocer que la educación va más allá de la migración de saberes hacia la migración de identidades, al compartir humanidades, fortalezas humanas, y convicciones humanas, pero también debilidades humanas e incertidumbres, dudas y angustias.

Loaiza-Robles (2009, p. 30) plantea en su obra que "El hacer didáctico al configurarse como acción que promueve la construcción de sentidos y significados", es decir, de darle significados a la realidad, nominar la realidad, darle rangos de valor a lo que existe, y a la forma de relacionarnos con lo que existe implica que la labor docente es una labor semántica, una labor de construcción de realidad, pero no de forma individual ni autocrática, sino como un ejercicio democrático (desde la perspectiva eminentemente social).

Esta mirada aborda la reflexión desde la propuesta teórica de didáctica no parametral (Quintar, 2008), en un escenario que será punto de partida propicio para el abordaje de esta noción pedagógica: los colectivos de maestros y maestras de la modalidad de educación a distancia de la Unidad Central del Valle del Cauca, UCEVA, lugar de encuentro de diferentes visiones y prácticas en torno al quehacer pedagógico y didáctico en la esfera de la formación de profesionales con licenciatura en educación. Es en la UCEVA, lugar de encuentro de saberes, lugar de encuentro vital para estudiantes y docentes, donde la educación a distancia se desarrolla como modalidad, pero también como una filosofía de lo educativo.

La didáctica no parametral, vista desde Quintar, es una nueva forma de comprensión de la realidad educativa desde el reconocimiento del estudiantado y del maestro y maestra como 
sujetos enteros, desde su enteridad, y en esa medida el proceso educativo debe ir más allá del simple reconocimiento de su multidimensionalidad, debe abordar esa mutidimensionalidad, intervenirla, construyendo desde lo cognoscitivo, lo afectivo, lo estético, lo ético y demás dimensiones del sujeto educable. El modelo educativo a distancia no es un escenario aparentemente propicio para esta construcción, un modelo donde la dificultad del encuentro desde lo humano solamente permite un intercambio de saberes, una estructura modélica de transacciones cognoscitivas.

Desde estas lógicas nos preguntamos, entonces, ¿qué emergencias - interacciones pueden surgir en la educación a distancia para construir escenarios de encuentro y enunciación del sujeto con otros sujetos y con su realidad, en articulación con didácticas no tradicionales?

Sobre esta pregunta articuladora, se elaboró la propuesta con el fin de hacer una acercamiento a la realidad de las prácticas didácticas del personal docente de la Licenciatura en Educación Básica con énfasis en Ciencias Sociales de la Uceva, en su modalidad a distancia.

\section{Metodología}

Esta investigación de orden cualitativo, descriptiva por su naturaleza comprensiva de un fenómeno educativo en contexto, se basó, fundamentalmente, en tres instrumentos: La entrevista en profundidad, los círculos de reflexión y el grupo focal.

El primer instrumento tuvo como finalidad identificar aspectos importantes del pensamiento y la práctica pedagógica de los maestros y maestras, a partir de la valoración de sus experiencias. Este instrumento nos permitió un acercamiento a la realidad de estos sujetos y de sus construcciones mentales frente a su hacer, sus prácticas cotidianas de aula.

El segundo instrumento fue un grupo focal con estudiantes, a fin de establecer la posibilidad del contraste, de la verificación por cruce de datos, a partir de sus experiencias y percepciones sobre las prácticas didácticas de sus docentes.

En el primer caso, las entrevistas a profundidad se realizaron a tres docentes, quienes se seleccionaron de forma intencionada, con base en dos criterios fundamentales:

- Que pertenecieran a cada uno de los tres momentos de formación de la licenciatura (formación básica, de profundización y de profesionalización)

- Que orientaran asignaturas cuyos contenidos aborden temas asociados con el proceso educativo: Didáctica, pedagogía, investigación, etc.

Para la aplicación de este instrumento se usó el mecanismo de grabación de audio.

Los resultados de las entrevistas a profundidad los mostraremos como metarrelatos de los datos obtenidos, y las aproximaciones conceptuales de los investigadores al respecto.

La docente N. 1, licenciada en Educación Física, con tres especializaciones en gerencia educativa, educación lúdica y docencia universitaria, y orientadora de asignaturas funamentalmente pedagógicas. En el proceso de entrevista manifiesta su cercanía en el uso de TIC como forma de aproximarse a sus estudiantes. Nos expresa que al mirarse en el 
espejo de su práctica didáctica reconoce como valioso el modelo constructivista, desde teorías como el aprendizaje significativo. Nos cuenta que la formación profesional es importante, pero es mucho más importante la relación interhumana entre docentes y estudiantes, y que su labor fundamental es de esta índole. Igualmente reconoce que la modalidad a distancia es limitante, pero que en manos del maestro está lograr romper esa distancia en la práctica.

El docente N. 2, licenciado, nos cuenta que la distancia es fundamentalmente una forma de enseñar, y que el proceso no puede ser limitante en la interacción con el estudiante. A partir de sus respuestas se puede inferir que este docente se asocia mucho más con lo conceptual que con la práxis educativa. Nos deja entrever que sus prácticas responden a modelos tradicionales, en los cuales se "dictan clase", pero sin mayores prácticas rupturales. Sin embargo, al ser consultado se identifica como constructivista.

El docente N. 3 también con formacion de licenciado y administrador, nos dice que usa la tecnología porque es una necesidad, pero no se aproxima a la importancia pedagógica de su uso. Igualmente sobre sus prácticas didacticas nos plantea que la formación didáctica sirve para "despertar más el conocimiento", lo que nos dice que no hay claridad conceptual. Nos dice que su estilo de enseñanza no es magistral, sino que es participativo. Manifiesta que considera necesaria una sana cercanía con los estudiantes, que no puede existir alejamiento porque no podrían lograrse avances en la construcción del conocimiento.

Un segundo instrumento fue el grupo focal. Este instrumento, aplicado en una sola sesión de trabajo, nos permitió contrastar la percepción de docentes y la de estudiantes sobre la práctica educativa, específicamente en las prácticas didácticas.

En el grupo focal participaron 26 estudiantes de diferentes semestres. Se les consultó a partir de un guion de preguntas semiestructuradas, con las cuales se buscó contrastar con los datos obtenidos en las entrevistas docentes. La sesión estuvo orientada por uno de los investigadores, mientras el otro documentaba el ejercicio. Se elaboró una guía de grupo de enfoque, que permitió darle organización a la sesión de trabajo.

Las preguntas guía del ejercicio fueron:

- ¿Cuál cree que es el estilo pedagógico de sus docentes?

- ¿Cómo son la relaciones entre ustedes y sus docentes en el aula?

- ¿Qué estrategias utilizan sus docentes para motivarlos a aprender?

- ¿Cómo son las evaluaciones que les hacen sus docentes'

- ¿Ha trabajado integradamente varias asignaturas en algún momento de su formación?

- ¿Cree que la modalidad a distancia afecta las interacciones con sus maestros?

A partir de estas premisas básicas se desarrolló un ejercicio de indagación, condensado en la tabla 1, que permitió identificar varios aspectos relevantes, a saber: 
Tabla 1

Estudiantes del grupo focal

\begin{tabular}{ll}
\hline Pregunta & Análisis de respuestas de estudiantes \\
\hline $\begin{array}{l}\text { ¿Cuál cree que es el estilo } \\
\text { pedagógico de sus } \\
\text { docentes? }\end{array}$ & $\begin{array}{l}\text { Coinciden en que el estilo en general de sus docentes es } \\
\text { bastante tradicional, y que hay pocas variantes respecto a la } \\
\text { clase magistral. Que se trabaja con base en talleres y trabajos, } \\
\text { pero la clase normalmente es magistral. }\end{array}$ \\
\hline $\begin{array}{l}\text { ¿Cómo son la relaciones } \\
\text { entre ustedes y sus docentes } \\
\text { en el aula? }\end{array}$ & $\begin{array}{l}\text { Hay diversidad de opiniones al respecto, sin embargo, hay una } \\
\text { coincidencia fundamental y es que en la mayoría de los casos } \\
\text { son relaciones estrictamente académicas, que con algunas } \\
\text { excepciones no ven en el maestro o maestra una persona } \\
\text { cercana, amiga, sino un profesor o profesora que da clases. }\end{array}$ \\
\hline $\begin{array}{l}\text { ¿Qué estrategias utilizan } \\
\text { sus docentes para } \\
\text { motivarles a aprender? }\end{array}$ & $\begin{array}{l}\text { Manifiestan que muy esporádicamente se hacen conversatorios } \\
\text { sobre un tema, o que se les de libertad de escoger un tema para } \\
\text { exponer. El uso de tecnologías es mínimo. }\end{array}$ \\
\hline $\begin{array}{l}\text { ¿Cómo son las evaluaciones } \\
\text { que les hacen sus docentes? }\end{array}$ & $\begin{array}{l}\text { Expresan que normalmente las evaluaciones son tradicionales, } \\
\text { la pregunta recurrente es por conceptos o por datos puntuales. } \\
\text { Existen casos de docentes que confrontan su capacidad de } \\
\text { crítica y opinión. }\end{array}$ \\
\hline $\begin{array}{l}\text { ¿Han trabajado } \\
\text { integradamente varias } \\
\text { asignaturas en algún } \\
\text { momento de su formación? }\end{array}$ & $\begin{array}{l}\text { Dicen que no, que cada asignatura se trabaja de forma } \\
\text { independiente. }\end{array}$ \\
\hline $\begin{array}{l}\text { ¿Cree que la modalidad } \\
\text { a distancia afecta las } \\
\text { interacciones con sus } \\
\text { maestros o maestras? }\end{array}$ & $\begin{array}{l}\text { Creen que sí, debido al poco tiempo que tienen con sus docentes, } \\
\text { a que los tiempos presenciales deben explotarse al máximo para } \\
\text { resolver dudas y programar actividades; entonces, la posibilidad } \\
\text { de que el personal docente conozca más a sus estudiantes o que } \\
\text { se convierta en una persona de su confianza es mínima. }\end{array}$ \\
\hline
\end{tabular}

Nota: Elaboración propia.

Un tercer instrumento (que se implementó a lo largo del primer semestre, 2014) es la herramienta que Estela Quintar denomina Circulos de reflexión, es decir, reuniones no estructuradas con docentes del programa de Licenciatura en Educación Básica con énfasis en Ciencias Sociales. Estas reuniones, tendrán como finalidad plantear temas de discusión sobre las prácticas docentes y su impacto en el proceso de enseñanza/aprendizaje. Se realizaron tres círculos, entre los meses de marzo y mayo de 2014.

La metodología que se utilizó en el desarrollo de esta actividad fue básica: Para cada sesión, los investigadores proponían un tema, a partir de un texto. El personal docente debía hacer la lectura previa y construir, a partir de esta, unas preguntas que tuvieran que 
ver con el ejercicio didáctico. Durante el desarrollo del círculo, un docente era elegido como moderador, y se proponían tres escenarios de análisis:

a. Qué dice el texto

b. Qué le aporta a mi saber didáctico

c. Cómo puedo usar estas ideas en la práctica en mis asignaturas.

Los textos analizados fueron:

- Modalidades pedagógicas, visibles e invisibles (Cap. 4), del libro Poder, educación y conciencia, de Basil Bernstein, CIDE, 1988.

- La educación como relación pedagógica para la resignificación del mundo de la vida, de Benjamin Berlanga, CESDER, 2003.

- Alteridades y pedagogías, de Carlos Skliar, en Educação \& Sociedade, 23(79), agosto, 2002.

- Si el otro no estuviera allí, de Carlos Skliar, en Educação \& Sociedade, 23(79), agosto, 2002.

- Didáctica no parametral, sendero hacia la desconización, de Quintar Ipecal, 2008.

Pretendimos que de estos círculos de reflexión salieran propuestas de nuevas prácticas didácticas, autoconstruidas por el colectivo docente, y que establecieran, como ruta articuladora, la ruptura con las prácticas tradicionales, a favor de alternativas creativas y desafiantes, que partan del reconocimiento de la condición humana del sujeto educable.

Si bien el objeto de esta investigación no llegó hasta el nivel de intervención, el desarrollo de los círculos si permitió que los grupos docentes puedieran, por ejemplo, intercambiar experiencias exitosas, y buscar formas alternativas para que esas experiencias exitosas se implementaran en diferentes asignaturas.

A modo de metanarración de las experiencias logradas en los círculos de reflexión, compartimos algunas de ellas:

El primer docente planteó un cambio de escenario, llevando a sus estudiantes a la zona verde de la Universidad, y planteándoles un conversatorio sobre el tema de clase. Si bien tenía preparada la clase, hizo el ejercicio de darles la palabra a sus estudiantes y al final hacer complemento, sin dar cátedra.

El segundo docente participante contó cómo elaboró una guía sobre una lectura, que incluía una dramatización del tema, lo cual consideró bastante interesante, pues en una asignatura aparentemente lineal como investigación, les permitió a sus estudiantes demostrar cómo harían un proceso de recolección de información en la vida real.

Un tercer docente ilustró de qué forma pidió a sus estudiantes que narraran experiencias personales de aprendizajes exitosos y de aprendizajes no exitosos en sus propios procesos de formación, lo cual les llevó a una discusión sobre educación, de gran valor pedagógico y cognitivo. 
El personal docente participante concluyó que esas primeras prácticas fueron valiosas porque sintieron que sus estudiantes se conectaron con los temas planteados, se involucraron y respondieron mucho más exitosamente a preguntas, de lo que se esperaba con la clase tradicional. Igualmente es relevante decir que los círculos de reflexión no pueden ser espacios de coyuntura, sino que deben elevarse a la categoría de encuentros permanentes, desde donde se alimentará la comprensión de la realidad, más allá de los modelos y las teorías, una mirada a los hábitus (Bourdieu) repensados por el grupo docente, implicó también cambios en los campus en los cuales se desempeña.

\section{Consideraciones finales}

Las experiencias educativas siempre son diferentes para las percepciones de estudiantes y maestros o maestras. Por eso las prácticas que para el personal docente son exitosas y amenas, para el estudiantado pueden ser simplemente cotidianas. Igualmente pasa con los modos de relación e interacción entre docentes y estudiantes.

Un ejercicio de reflexión permanente sobre la práctica; sobre la forma como los procesos didácticos se transforman en interacciones humanas; como las transmisiones de saberes migran hacia la construcción social de ciudadanía, de formación cultural, axiológica y científica, permiten al personal docente hacer mayores impactos en sus estudiantes, contribuyendo a la formación de personas críticas, reflexivas, capaces de asumir posiciones frente a los retos de la contemporaneidad.

Solo una práctica educativa que rompa los ejes tradicionales del transmisionismo pedagógico podrá, en principio, aportar a una educación más centrada el reconocimiento de la condición humana, y en la construcción de interacciones humanas más asertivas y éticas.

Se pudo concluir que, si bien las metódicas de la modalidad de educación a distancia en la UCEVA no excluyen completamente la presencialidad, es necesario repensar la forma como esos momentos de encuentro se convierten en escenarios de enunciación en los cuales el ámbito humano cobre relevancia, más allá del simple compartir de contenidos.

El uso de la tecnología, si bien no se ha masificado en el programa objeto de estudio, aún no contribuye a ser canal de comunicación permanente entre docentes y estudiantes, para mitigar las distancias que propicia una dinámica académica con encuentros presenciales tan escasos.

Es importante hacer un ejercicio permanente de reflexión sobre las metódicas didácticas del programa, y así migrar de un modelo transmisionista, basado en contenidos, a un modelo educacional basado en las interacciones humanas y en la formación de sujetos integrales para la sociedad.

\section{Referencias}

Ausubel, D. (1981). Psicología educativa, un punto de vista cognoscitivo. México: Trillas.

Buitrago, L. S., Torres, L. V. y Hernández, R. M. (2009). La secuencia didáctica en los proyectos de aula, un espacio de interrelación entre docentes y contenido de enseñanza. Bogotá: Pontifica Universidad Javeriana. 
De Zubiría, J. (2006). Los modelos pedagógicos, hacia una pedagogía dialogante. Bogotá: Magisterio.

García, L. (mayo-agosto, 1991). Un concepto integrador de enseñanza a distancia. Radio y Educación de Adultos. 17, 3-6. Recuperado de http://www2.uned.es/catedraunesco-ead/ articulos/1991/un\%20concepto\%20integrador\%20de\%20ensenanza\%20a\%20distancia.pdf

Gómez, M. V. (2001). Paulo Freire y la agenda de educación latinoamericana en el siglo XXI. México: Clacso.

Loaiza-Robles, F. (2009). didáctica no parametral, diálogos para repensar la educación ambiental. Manizalez: Cinde.

Quintar, E. B. (1998). La enseñanza como puente a la vida. México: IPECAL.

Quintar, E. B. (2008). Didáctica no parametral, sendero hacia la descolonización. México: Ipecal.

Saa, A. L. y Garcés, A. M. (2012). Resignificación del sujeto mediante didácticas no parametrales para posibilitar la reafirmación de la condición afrodescendiente en la institución educativa Termarit, del municipio de Buenaventura. Manizalez: UCM.

Zambrano-Leal, A. (2001). La mirada del sujeto educable, la pedagogía y la cuestión del otro. Cali: Fundacióin para la filosofía en Colombia.

Zemelman, H. (2006). Sujeto, existencia y potencia. México: Anthropos. 